\title{
Pancreatoscopy-guided laser lithotripsy in a patient with difficult ductal stone
}

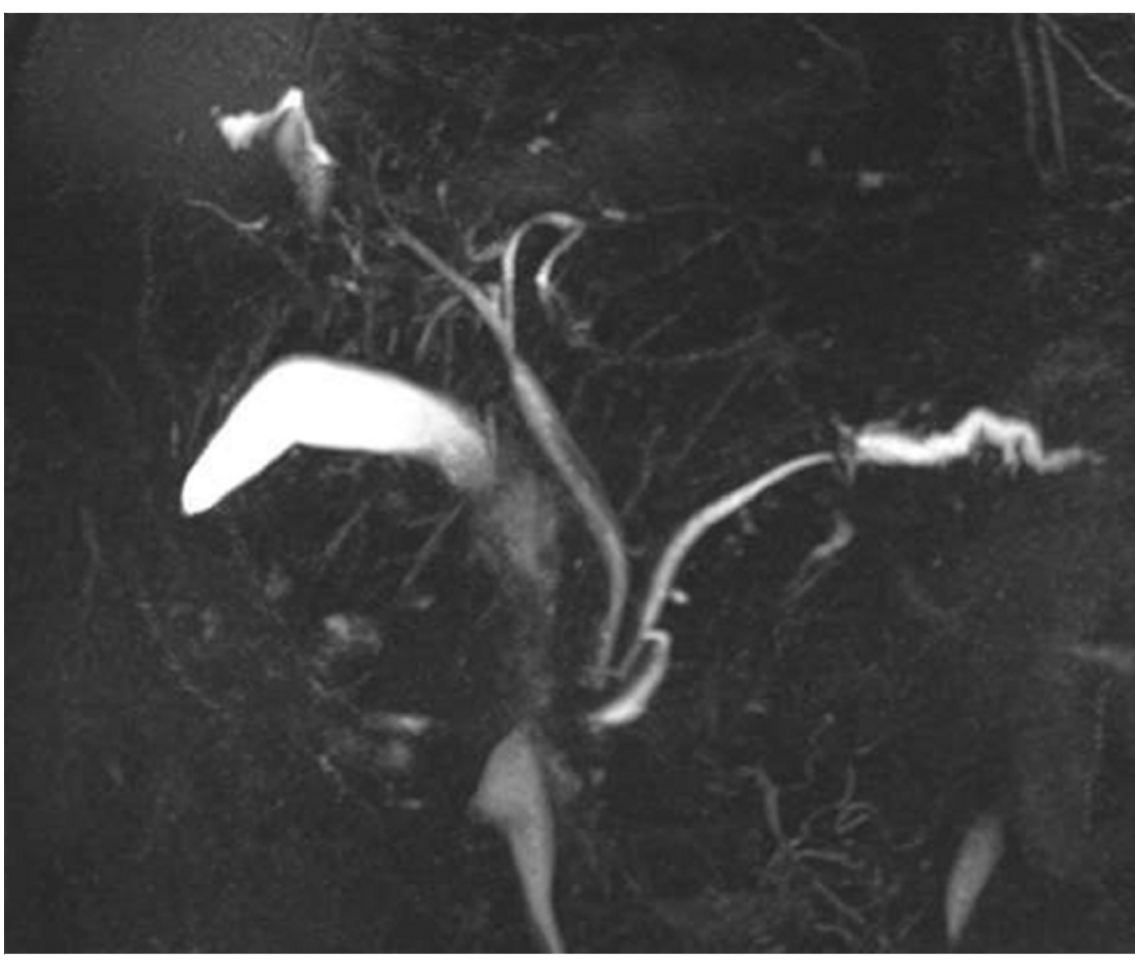

- Fig. 1 Magnetic resonance cholangiopancreatography, showing an abrupt stop at the pancreatic body, followed by major dilation of the remaining body and tail.

A 63-year-old man with heavy alcohol consumption was referred to our institution for upper abdominal pain, weight loss, and a computed tomography scan showing signs of chronic pancreatitis (parenchymal calcifications and atrophy of the pancreatic body/tail).

A magnetic resonance cholangiopancreatography was performed, showing Wirsung dilation, namely of the tail, and an abrupt stop in the pancreatic body of unknown cause ( $\mathbf{F i g} \mathbf{1}$ ). Endoscopic ultrasound revealed an intraductal stone in the pancreatic body $(7.4 \mathrm{~mm})$, and a dilated Wirsung in the body and tail ( $\vee$ Fig.2). The patient underwent endoscopic retrograde cholangiopancreatography (ERCP).

Pancreatography showed an irregular Wirsung contour in the head and irregularity in the body-tail transition, suggestive of an intraductal stone (> Fig.3).
Pancreatic sphincterotomy was performed, and the calculus was crossed with the guidewire, but it was impossible to cross it with a 6-mm dilation balloon. After hydrostatic balloon dilation of the pancreatic head (up to $6 \mathrm{~mm}$ ), a pancreatoscope (Spyglass Direct Visualization System; Boston Scientific, Marlborough, Massachusetts, USA) was advanced over a 0.025 -inch guidewire to reach a large intraductal stone of $7-8 \mathrm{~mm}$ in size ( Fig.4, $\vee$ Video 1). After targeting the stone, laser bursts (Holmium laser, Auriga $\mathrm{XL}$; Boston Scientific) of less than 5 seconds were delivered through the aqueous medium using a 365- $\mu$ m diameter fiber (energy level $1200 \mathrm{~m}$ ); frequency of $12 \mathrm{~Hz}$ ). After stone fragmentation, ductal clearance was achieved with an 8.5-mm extraction balloon. Two pancreatic stents $(12 \mathrm{~cm}, 7 \mathrm{Fr})$ were placed.

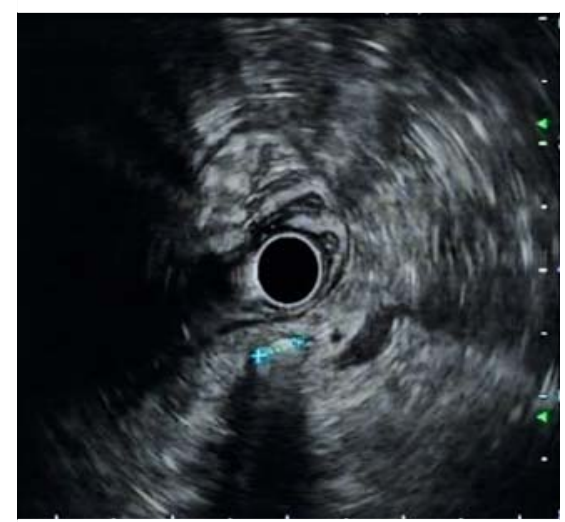

- Fig. 2 Endoscopic ultrasound revealed a large intraductal stone in the pancreatic body $(7.4 \mathrm{~mm})$, and a dilated Wirsung in the body and tail.

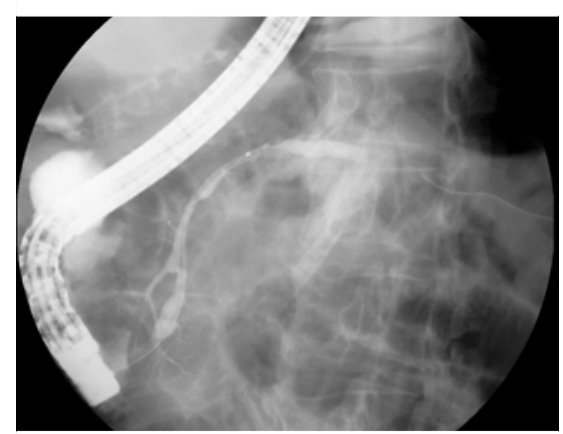

Fig. 3 Pancreatography showing irregularity in the proximal body, suggesting a large intraductal stone.

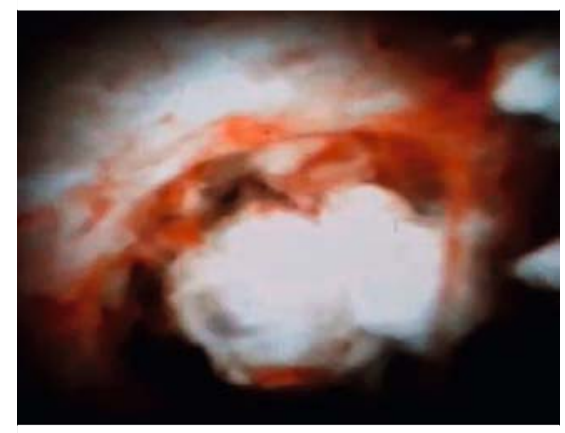

> Fig. 4 Pancreatoscopy image showing a large intraductal stone impacted on a main duct stricture. 

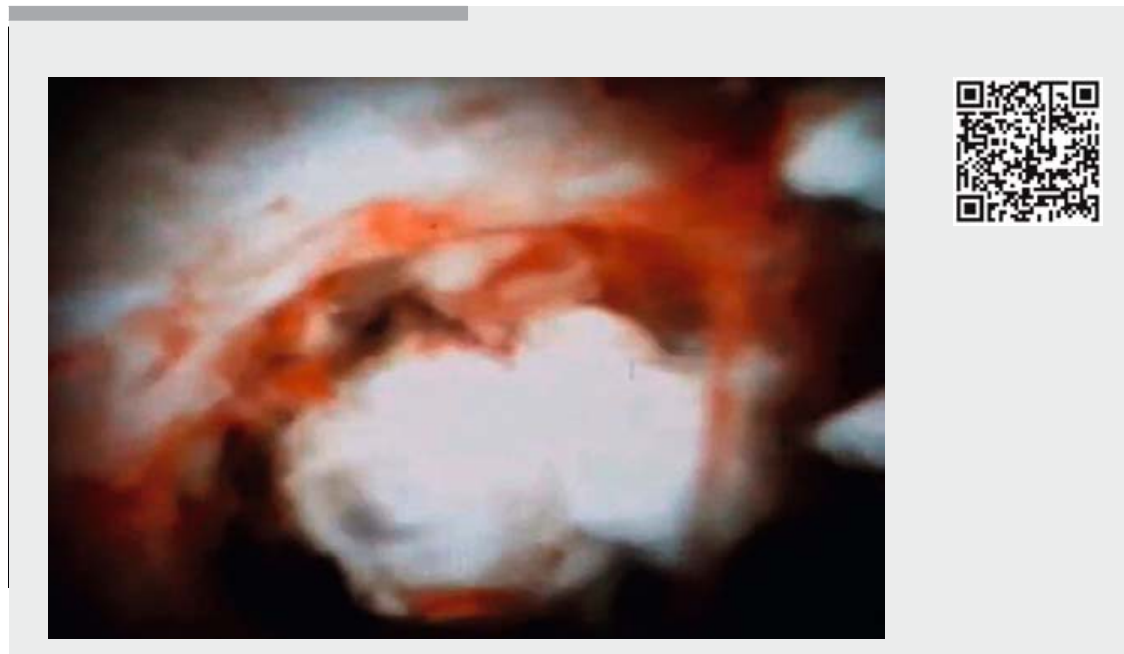

Video 1 Intraductal pancreatoscopy with holmium laser lithotripsy was performed until complete stone fragmentation was achieved.

At follow-up 3 months later, repeat ERCP showed frank improvement of the head stricture, without filling defects in the remaining Wirsung ( $>$ Fig.5). The patient remained asymptomatic during followup ( 6 months) without further interventions.

Published experience is limited, but pancreatoscopy-guided laser lithotripsy for calcific chronic pancreatitis is a promising technique that can be used as a supplementary approach to extracorporeal shock wave lithotripsy or as a single modality in a small number of stones obstructing the main pancreatic duct [1-4].

Endoscopy_UCTN_Code_TTT_1AR_2AI

\section{Competing interests}

Jorge Canena is a consultant for Boston Scientific but did not receive any financial arrangements for this research or any assistance with manuscript preparation.

\section{The authors}

Gonçalo Alexandrino, Luís Lourenço, Catarina G. Rodrigues, David Horta, Jorge Reis, Jorge

\section{Canena}

Department of Gastroenterology, Hospital Prof. Doutor Fernando Fonseca, Amadora, Portugal

\section{Corresponding author}

\section{Gonçalo Alexandrino, MD}

Department of Gastroenterology, Hospital

Prof. Doutor Fernando Fonseca, IC 19

Amadora 2720-276, Portugal

Fax: +351-21-4345566

goncaloalexandrino@gmail.com

\section{References}

[1] Attwell AR, Patel S, Kahaleh M et al. ERCP with per-oral pancreatoscopy-guided laser lithotripsy for calcific chronic pancreatitis: a multicenter U.S. experience. Gastrointest Endosc 2015; 82: $311-318$

[2] Shah J. Innovations in intraductal endoscopy: cholangioscopy and pancreatoscopy. Gastrointest Endosc Clin N Am 2015; 25: 779-792

[3] Ang TL. Chronic pancreatitis with pancreatic duct stricture and calculi treated by fully covered self-expandable metal stent placement and intraductal pancreatoscopy-guided laser lithotripsy. Endoscopy 2017; 49: E145-E146

[4] Maydeo A, Kwek BE, Bhandari S et al. Singleoperator cholangioscopy-guided laser lithotripsy in patients with difficult biliary and pancreatic ductal stones (with videos). Gastointestin Endosc 2011; 74: 1308 - 1314

\section{Bibliography}

DOI https://doi.org/10.1055/a-0574-2278

Published online: 8.3.2018

Endoscopy 2018; 50: E130-E131

(c) Georg Thieme Verlag KG

Stuttgart · New York

ISSN 0013-726X

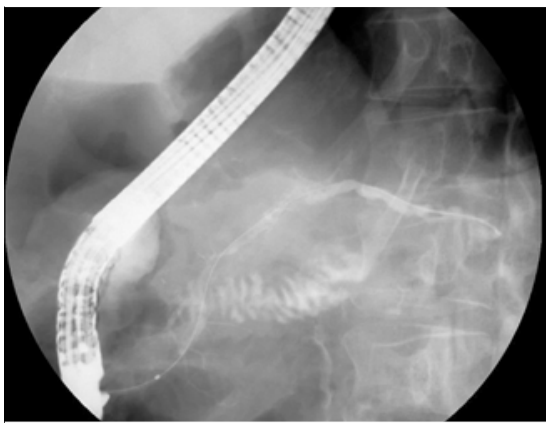

- Fig. 5 Pancreatogram image showing resolution of the pancreatic stricture after successful endotherapy.

\section{ENDOSCOPY E-VIDEOS}

https://eref.thieme.de/e-videos

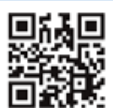

Endoscopy E-Videos is a free access online section, reporting on interesting cases and new techniques in gastroenterological endoscopy. All papers include a high quality video and all contributions are freely accessible online.

This section has its own submission website at

https://mc.manuscriptcentral.com/e-videos

\section{CORRECTION}

Alexandrino G, Lourenço L, Rodrigues CG et al. Pancreatoscopy-guided laser lithotripsy in a patient with difficult ductal stone.

Endoscopy 2018,

doi:10.1055/a-0574-2278

In the above mentioned article one author was missing in the authors' list. Correct is: Gonçalo Alexandrino, Luís Lourenço, Catarina G. Rodrigues, David Horta, Jorge Reis, Jorge Canena.

This was corrected in the online version on April 6, 2018. 